\title{
Integrating Competition Strategy to QFD for Technical Design
}

\author{
Yai Hsiung \\ Pao-Yuan Chang* \\ Dept. of Information Management, Ta Hwa Institute of Technology, Hsinchu, Taiwan, R.O.C. \\ Dept. of Information Management, Overseas Chinese Institute of Technology, Taichung, Taiwan, R.O.C.
}

\begin{abstract}
Quality Function Deployment (QFD) is an approach to translate customer requirements into technical requirements for a product or service. The traditional QFD provides a fundamental analysis, but it is still inconvenient when decision making in technical design should address business objection. The competition strategies and the resource allocation for this improvement planning are essential analysis but unfortunately they are neglected by the traditional QFD. This paper presents an innovative QFD approach, which takes competition strategies and the resource allocation into account to provide a more precise procedure for identifying the engineering design characteristics. We also translate QFD into a linear programming model for determining the resource allocation optimization.
\end{abstract}

Keywords: QFD, Resource Allocation, Linear programming

\section{Introduction}

Quality Function Deployment (QFD) is a popular tool for identifying the strategy of technical requirements for a product or service. The concept of QFD is to integrate customer requirements into technical (design or manufacturing) items by a set of related matrices. QFD is a useful approach for planning the specifications of a product or service,

*Corresponding author. Tel: 1-886-4-27016855 2132 E-mail: pychang@ocit.edu.tw because its property of being customer oriented ensures the magnitude of customer required to be translated into the process aspects of the product.

Unfortunately, there still exists some limitation when one tries to plan the product strategy by the QFD approach. The competitive evaluation of customer requirements does not provide sufficient information for the analysis of technical specification. The competitive evaluation based on customer requirements is important assessment information for marketing planning, and this information should be translated into the competitive evaluation of technical requirements. In fact, the traditional QFD procedure includes both the competitive evaluation based on customer and technique requirements, but it treats the two evaluations as separate analyses. In our opinion, we believe that there is some relationship between the two competitive evaluations, and therefore the two evaluations should not be analyzed separately. Moreover, the competitive evaluation based on technical requirements is hard to be obtained because it involves sensitive commercial information. Besides, without the analysis of resource allocation, QFD can not decide which technical specifications should be improved if the financial budget is limited. Finally, for technique strategy, a decision maker is interested to know how to improve the technical specifications to become the winner (or achieve the competitive target) to competitors, but QFD does not provide such insight analysis.

This paper presents an innovative approach for 
planning the product specification, which integrates competitive strategy into QFD, and collaborates with linear programming to provide a more precise tool for decision making.

\section{The previous works}

The related research on QFD is plentiful, but due to the limitations of pages, this study gives brief reviews in the followings. QFD approach was proposed in 1970s for product quality improvement especially in mobile manufacturing. Since then, QFD is broadly applied in new product development [1]. QFD is a decision making tool, which translates the magnitude of quality into a quantity scale for evaluation. Locascio [2] introduces a multi-attribute theory into QFD to determine the technical attributes of a product. Moskowitz and Kim [3] use statistical regression method for determining the function between customer and technical requirements. Kim et al. [4] develop a rule-based scheme to enhance the function of QFD. To reflect the imprecise expression of decision making of humans, a fuzzy approach is integrated into QFD [5, 6, 7], which defines linguistic terms and utilizes fuzzy operators to decide the engineering specifications.

This paper proposes a revised QFD approach, which determines the optimal resource allocation (the optimal design of technique) under limited resources. We develop a revised QFD procedure, and then translate the problem into linear programming. The software "SOLVER" executes the final solution.

\section{The proposed QFD}

\section{Indices:}

$i$ : customer requirements $(i=1, \ldots, m)$

$j$ : technical requirements $(j=1, \ldots, n)$

$k$ : main competitors $(k=1, \ldots, g)$, where $k=1$ represents the company to be planned.
The related matrices are defined as follows.

(1) The relationship matrix between customer and technical requirements

$$
R=\left[\begin{array}{ccc}
r_{11} & \cdots & r_{1 n} \\
\vdots & r_{i j} & \vdots \\
r_{m 1} & \cdots & r_{m n}
\end{array}\right]
$$

(2) The importance matrix (vector) of customer requirements

$$
W_{0}=\left[w_{1}, \ldots, w_{i}, \ldots, w_{m}\right]
$$

(3) The competitive evaluation matrix-customer for performance of customer requirements

$$
U=\left[\begin{array}{ccc}
u_{11} & \cdots & u_{1 m} \\
\vdots & u_{k i} & \vdots \\
& & \\
u_{g 1} & \cdots & u_{g m}
\end{array}\right]
$$

(4) The competitive evaluation matrix-technique for performance of technical requirements

$$
T=\left[\begin{array}{ccc}
t_{11} & \cdots & t_{1 n} \\
\vdots & t_{k j} & \vdots \\
t_{g 1} & \cdots & t_{g n}
\end{array}\right]
$$

(4) The correlation matrix between technical requirements

$$
L=\left[\begin{array}{ccc}
l_{11} & \cdots & r_{1 n} \\
\vdots & l_{j j} & \vdots \\
r_{n 1} & \cdots & r_{n n}
\end{array}\right]=\left[\begin{array}{ccc}
1 & \cdots & r_{1 n} \\
& 1 & \vdots \\
& & \\
& & 1
\end{array}\right]
$$

(5) The cost matrix (vector) of technical requirement improvement

$$
C=\left[c_{1}, \ldots, c_{j}, \ldots, c_{n}\right]
$$

\section{The procedure of the proposed QFD}

The procedure of the proposed QFD is as follows.

(1) Define relationship matrix $R$

(2) Obtain the competitive evaluation 
matrix-customer $U$

(3) Translate the competitive evaluation matrix-customer $U$ to the performance matrix-technical $T$ by the following equation.

$T=U \cdot R$

(4) Let $x_{j}$ represent the resource to be added for technical requirement $j$ for improvement.

Let $y_{j}$ represents the performance improved in technical requirement $j$ if resource $x_{j}$ is added. The relation between $y_{j}$ and $x_{j}$ can be interpreted as output and input. The relation function can be assumed as equation (2).

$$
y_{j}=f_{j}\left(x_{j}\right)
$$

where function $f(\cdot)$ denotes the relationship between the added resource and its improved performance.

Let $T_{1}$ represent the competitive evaluation matrix of technical requirements after adding the new resource.

where $T_{1}=\left[\begin{array}{ccccc}t_{11}+y_{1} & \cdots & t_{1 j}+y_{j} & \cdots & t_{1 n}+y_{n} \\ \vdots & & t_{k j} & & \vdots \\ & & & & \\ t_{g 1} & & \cdots & & t_{g n}\end{array}\right]$

The evaluation based on customer requirements can be improved after adding the new resources. Let $U_{1}$ denote the new competitive evaluation matrix-customer.
Let $U_{1}=\left[\begin{array}{ccccc}u_{11}+z_{1} & \cdots & u_{1 i}+z_{i} & \cdots & u_{1 m}+z_{m} \\ \vdots & & u_{k i} & & \vdots \\ & & & & \\ u_{g 1} & & \cdots & & u_{g m}\end{array}\right]$

where $Z_{i}$ represents the increased performance of customer requirements after adding technical resources.

$U_{1}$ can be obtained by the following equation.

$$
\begin{gathered}
T_{1}=U_{1} \cdot R \\
\Rightarrow U_{1}=R \cdot T_{1}^{-1}
\end{gathered}
$$

It is noted that $Z_{i}$ can be converted into variable $x_{j}$ by equations (2) and (3).

(5) Model constraints equation of problem.

Let $A$ represents the difference of customer requirements matrix between the company and competitors

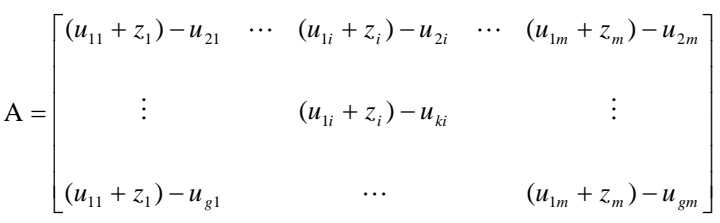

To ensure the company be the winner to competitors, equation (4) should be developed.

$$
W \cdot A^{T} \geq 0
$$

Equation (4) can be written as (5).

$\Rightarrow\left[w_{1}, \ldots, w_{i}, \ldots, w_{m}\right] \cdot\left[\begin{array}{ccc}\left(u_{11}+z_{1}\right)-u_{21} & \cdots & \left.u_{11}+z_{1}\right)-u_{g 1} \\ \vdots & \vdots & \vdots \\ \left(u_{1 m}+z_{m}\right)-u_{2 m} & \cdots & \left(u_{1 m}+z_{m}\right)-u_{g m}\end{array}\right] \geq\left[\begin{array}{c}0 \\ \vdots \\ 0\end{array}\right]$

(6) Model the objective function. 
The goal of objective function is to minimize the cost of required resources. That is

$$
\operatorname{Min} Z=C \cdot X^{T}
$$

(7) Problem solving

The QFD problem is translated as the following mathematic program.

$$
\begin{aligned}
& \text { Min } Z=C \cdot X^{T} \\
& W \cdot A^{T} \geq 0 \\
& X \geq 0
\end{aligned}
$$

The above problem is a linear programming problem and it can be solved by mathematics software easily.

\section{Conclusion}

Technical design is a critical task for manufacturing strategy of products. The traditional QFD provides a fundamental analysis for strategy of technical design, but still neglects some important factors to address business objections. The factors of competition strategies and the required cost are essential for improvement planning and should be involved in the QFD analysis. In this paper, we proposed an innovative scheme for QFD, in which competition strategies and resource allocation information are concerned. We translated the QFD analysis into a linear programming model for determining the resource allocation optimization.

\section{References}

[1] B. Prasad, "Review of QFD and Related
Deployment Techniques," Journal of Manufacturing Systems, 17(3), pp. 221-234, 1998.

[2] A. Locascio and D. L. Thurston, "Multi-attribute Design Optimization with Quality Function Deployment," $2^{\text {nd }}$ Industrial Engineering Research Conference Proceedings, LA, USA, pp. 82-86, 1993.

[3] H. Moskowitz and K. J. Kim, “QFD Optimizer: A Novice Friendly Quality Function Deployment Decision Support System for Optimizing product Designs, ” Computers Industrial Engineering, 3, pp. 641-655, 1997.

[4] J. K. Kim, C. H. Han and S. H. Choi, “A Knowledge-Base Approach to The Quality Function Deployment," Computers and Industrial Engineering, 35(1-2), pp. 233-236, 1998.

[5] Y. Q. Yang, S. q. Wang, M. Dulaimi and S. P. Low, “A Fuzzy Quality Function Deployment System for Buildable Design Decision-Makings,” Automation in Construction, pp. 381-393, 2003.

[6] L. H. Chen and M. C. Weng, “A Fuzzy Model for Exploiting Quality Function Deployment," Mathematical and Computer Modelling, 38, pp. 559-570, 2003.

[7] Y. K. Funk, Y. Chen and J. Tang, "Estimating the Functional Relationships for Quality Function Deployment under Uncertainties,” Fuzzy Sets and Systems, 157, pp.98-120, 2006. 